\title{
Criterios de evaluación de impacto ambiental en el sector minero
}

\section{RESUMEN}

El Perú es un país minero por excelencia, por lo cual las empresas mineras explotan estos recursos. La realización de estas actividades, genera un impacto en el ambiente que concierne a la flora, fauna, aspectos socioeconómicos, etc. Es por ello que el Ministerio de ambiente evalúa el impacto ambiental mediante parámetros como: extensión, naturaleza, que deben dar como resultado final si es viable 0 no continuar con el proyecto minero. El proceso de evaluación de impacto ambiental puede durar meses, incluso años. El presente artículo propone los criterios de evaluación de impacto ambiental en el sector minero tal que sirvan de apoyo a la toma de decisiones.

Palabras clave: indicador de importancia, impacto ambiental

Criteria for evaluating the environmental IMPACT ON THE MINING SECTOR

\section{ABSTRACT}

Peru is a mining country par excellence, by which many mining companies exploit these resources. In making these activities generates impact the environment concerning the flora, fauna, socio-economics, etc. That is why the Ministry of Environment evaluates the environmental impact by parameters such as size, nature, which must give the final result if it is viable or not to continue with the mining project. The process of evaluation of environmental impact can last for months, even years. This article proposes criteria for environmental impact assessment in the mining sector may serve to support decision making.

Keywords: environmental impact, indicator of importance

\section{INTRODUCCIÓN}

Desde la aparición de la humanidad se ha establecido relaciones con el medio ambiente que puede ser afectada de menor o mayor nivel, las actividades humanas han dado como resultado la contaminación ambiental, como por ejemplo cuando nuestros antepasados establecieron el sedentarismo, empezaron con la tala de árboles, sin embargo esta ha sido claramente visto a partir de la Revolución industrial [web 01], al querer automatizar las actividades humanas han hecho uso de diferentes métodos que van en contra del medio ambiente, además de la urbanización, donde las personas han invadido zonas que inicialmente resultaban ser áreas verdes.

En el Perú la institución encargada del cuidado del medio ambiente es el Ministerio del Ambiente [web 01]. Siendo el Perú un país minero es pertinente establecer los criterios apropiados para la evaluación del impacto ambiental incluso antes de iniciar las operaciones de extracción.

\section{Objetivo general}

- Establecer criterios de evaluación de impacto ambiental en el sector minero tal que permita establecer medidas correctivas cuando aparezca el problema.

\section{Objetivos específicos}

- Identificar los problemas generados por los químicos utilizados por la actividad minera, a fin de establecer criterios que mitiguen el impacto ambiental.

- Generar la evaluación ambiental que utilice todas las normas, leyes y reglamentos que rige el Ministerio del ambiente para que dicha institución apruebe la evaluación ambiental.

\section{Problemas específicos}

Los efectos negativos en la extracción de diversos minerales a causa del uso de sustancias toxicas que contaminan tanto el aire como el suelo, involucran no solo el factor ambiental, sino el social, que también repercute en enfermedades propias de dichas sustancias

* Ingeniero de Sistemas e Informática, UNMSM. E-mail: diana.soriano.p@gmail.com

** Licenciada en computación, Profesora de la facultad de Ingeniería de sistemas e informática, UNMSM. E-mail: mruizr@unmsm.edu.pe,merruri@hotmail.com

*** Magíster en informática, Docente Principal UNMSM. E-mail: edgar.ruiz@industrial.unmsm.pe 
La omisión de algunos reglamentos, normas, leyes establecidas para el cumplimiento de dicha evaluación de impacto ambiental que estipula el Ministerio del ambiente en nuestro país. [web 02]

\section{Justificación}

Es necesario contar con criterios de Evaluación del Impacto Ambiental de los Proyectos de Inversión en el sector minero, para una mejor agilización en la toma de decisiones de dicha evaluación, así como para el otorgamiento del permiso si se contempla dichos criterios.

\section{FUNDAMENTACION TEÓRICA}

\subsection{Medioambiente}

\subsubsection{Evaluación de Impacto Ambiental}

\section{Medioambientes}

Es el conjunto de factores físicos, químicos, biológicos, sociales, culturales, estéticos y económicos capaces de causar efectos entre sí, con el individuo y con la comunidad en la que vive, determinando su forma, carácter, relación y supervivencia.

\section{Medio físico o natural}

Sistema constituido por los elementos y proceso del ambiente natural tal como encontramos en la actualidad y sus relaciones con la población.

Se proyecta en tres subsistemas:

- Medio Inerte o Medio Físico propiamente dicho: Aire, Tierra y Agua.

- Medio Biótico: Flora y Fauna.

- Medio Perceptual: Unidades de paisaje (cuencas visuales, valles y vistas).

\section{Medio Socio-económico}

Sistema constituido por las estructuras y condiciones sociales, histórico culturales y económicas en general, de las comunidades humanas o de la población de un área determinada.

\section{Factores ambientales}

Son los distintos componentes del Medio Ambiente entre los cuales se desarrolla la vida en nuestro planeta. Pueden ser modificados por las acciones humanas, en ocasiones, provocando grandes alteraciones que pueden ocasionar graves problemas generalmente difíciles de valorar.

Los organismos competentes de la CEE consideran estos factores ambientales: El hombre, la flora y la fauna; el suelo, el agua, el aire, el clima y el paisaje; las interacciones entre los anteriores; los bienes materiales y el patrimonio cultural.

\subsubsection{Impacto ambiental}

La alteración, modificación o cambio en el ambiente, o en alguno de sus componentes de cierta magnitud y complejidad o producido por los efectos de la acción o actividad humana. Esta acción puede ser un proyecto de ingeniería, un programa, un plan, o una disposición administrativo-jurídica con implicaciones ambientales.

Debe quedar explícito, sin embargo, que el término impacto no implica negatividad, ya que éste puede ser tanto positivo como negativo.

\section{Evaluación de Impacto Ambiental (EIA)}

Es un procedimiento jurídico-técnico-administrativo que tiene por objeto la identificación, predicción e interpretación de los impactos ambientales que un proyecto o actividad produciría en caso de ser ejecutado; así como la prevención, corrección y valoración de los mismos. Todo ello con el fin de ser aceptado, modificado o rechazado por parte de las distintas Administraciones Públicas competentes.

\section{Estudio del Impacto Ambiental (EsIA)}

Se puede definir como el estudio técnico, de carácter interdisciplinario, que incorporado en el procedimiento de la EIA, está destinado a predecir, identificar, valorar y corregir, las consecuencias o efectos ambientales que determinadas acciones pueden causar sobre la calidad de vida del hombre y su entorno. Es un documento técnico que debe presentar el titular del proyecto y sobre la base del cual se produce la Declaración o Estimación de Impacto Ambiental.

Se trata de presentar la realidad objetiva, para conocer en qué medida repercutirá sobre el entorno la puesta en marcha de un proyecto, obra o actividad y con ello, la magnitud de la presión que dicho entorno deberá soportar.

\section{Valoración del Impacto Ambiental (VIA)}

Forma parte de la última fase del EIA y consiste en transformar los impactos medidos en unidades heterogéneas, a unidades homogéneas de impacto ambiental, de forma que permita comparar varias alternativas diferentes de un mismo proyecto y también de proyectos distintos.

\section{Indicador de Impacto Ambiental}

Factor ambiental cuyo cambio proporciona la medida de la magnitud del impacto, al menos en su aspecto cualitativo y también, si es posible en el cuantitativo. 


\section{Importancia de un Impacto}

Valoración que nos da una especie de ponderación del impacto. Expresa la importancia del efecto de una acción sobre un factor ambiental.

\section{Concepto de Impacto Ambiental}

El término de impacto se aplica a la alteración que introduce una actividad humana en el entorno; este último concepto identifica la parte del medio ambiente afectada por la actividad, o más ampliamente, que interacciona con ella. No se suele aplicar el término impacto a las alteraciones ambientales producidas por fenómenos naturales, como los daños causados por una tormenta. Por tanto el impacto ambiental se origina en una acción humana y se manifiesta según tres facetas sucesivas:

- La modificación de alguno de los factores ambientales 0 del conjunto del sistema ambiental.

- La modificación del valor del factor alterado o del conjunto del sistema ambiental.

- La interpretación o significado ambiental de dichas modificaciones, y en último término, para la salud y el bienestar humano. Esta tercera faceta está íntimamente relacionada con la anterior ya que el significado ambiental de la modificación del valor no puede desligarse del significado ambiental del valor de que se parte.

\section{Concepto de Evaluación de Impacto Ambiental}

La Evaluación de Impacto Ambiental es, ante todo y como su propio nombre indica, una valoración de los impactos que se producen sobre el medio ambiente por un determinado proyecto [Web 01]. Ésta nunca puede ser objetiva, ya que tiene siempre connotaciones subjetivas debido a que la referencia es la calidad ambiental, un concepto subjetivo. La Ciencia, o una visión puramente objetiva del ambiente, aunque puede proporcionar las herramientas necesarias para justificar un argumento, no sirve para realizar la valoración en sí, ya que los factores éticos se escapan del ámbito científico y por lo tanto, no pueden considerarse objetivos, aunque no por ello deban de ser arbitrarios.

En todo el proceso de evaluación de impacto ambiental se persigue un objetivo claro: valorar adecuadamente las acciones sobre el entorno de forma que puedan encuadrarse dentro del proceso de toma de decisiones y poder decidir si la realización de un proyecto determinado es o no aceptable desde un punto de vista ambiental.

\subsection{Minería}

La minería industrial es el proceso de extracción, explotación y aprovechamiento de minerales que se hallan sobre la superficie terrestre con fines comerciales. [Web 03] Si se extraen metales de los minerales extraídos, la técnica de la minería se refina originando diferentes tipos de técnicas metalúrgicas. La minería es una de las actividades más antiguas desarrollada por el hombre. Al dominar el fuego, el hombre desarrolló técnicas que le permitieron procesar y elaborar los metales. Así comenzó a gestarse la primera revolución tecnológica histórica: La extracción de los minerales.

\section{Etapas del proceso Minero:}

Exploración: Detección de yacimientos naturales.

Explotación: Extracción de minerales.

- Análisis de propiedades físicas y químicas.

- Si puede utilizarse sin alteración de su sustancia, entra en etapa de Abastecimiento.

- Si puede destruirse para generar un producto nuevo, sigue la cadena de producción hasta entrar en etapa de Abastecimiento (depende del producto a generar).

Abastecimiento: se inicia la cadena de proceso productivo que lo llevará al consumidor final.

\section{METOdOLOGÍAS PARA LA EVALUACIÓN DE IMPACTO AMBIENTAL}

Existen diferentes métodos, metodologías y procedimientos para evaluar los impactos ambientales, ya sea para evaluar el estado del Medio Ambiente en general o para evaluar específicamente alguno de sus factores.

Las características deseables en las metodologías que se adopten para la evaluación del impacto ambiental, comprenden los siguientes aspectos [01]:

- Deben ser adecuados para las tareas de identificación de impactos y comparación de opciones.

- Ser lo suficientemente independiente de los puntos de vista del personal del equipo evaluador.

- Ser económicos en términos de costes, requerimientos de datos, tiempo de aplicación, etc.

A continuación se presentan brevemente algunas de las metodologías de evaluación de impacto ambiental más utilizadas. 


\subsection{Listas de Chequeo}

Es un método muy simple, por lo que es de uso frecuente. Se utiliza normalmente para una evaluación preliminar o para llamar la atención sobre impactos importantes.

Hay muchas variedades de listas de chequeo. Típicamente, la lista de chequeo contiene una serie de puntos, asuntos de impacto o cuestiones que el usuario atenderá o contestará como parte del estudio de impacto. Tales listas de chequeo representan recordatorios útiles para identificar impactos y proporcionar una base sistemática y reproducible para el proceso de EIA. Pueden clasificarse en cuatro tipos:

- Simples: Analizan factores o parámetros sin ser estos valorados o interpretados.

- Descriptivas: Analizan factores o parámetros y presentan la información referida a los efectos sobre el medio.

- De verificación y escala: Incluyen, además de lo anterior, una escala de carácter subjetivo para la valoración de los efectos ambientales.

- De verificación, escala y ponderación: Añaden a las anteriores unas relaciones de ponderación de factores en la escala de valoración.

\subsection{Métodos Matriciales}

Los métodos matriciales son técnicas bidimensionales que relacionan acciones con factores ambientales; y son básicamente de identificación. Los métodos matriciales, también denominados matrices interactivas causa-efecto, fueron los primeros en ser desarrollados para la EIA. La modalidad más simple de estas matrices muestra las acciones del proyecto en un eje y los factores del medio a lo largo del otro. [02], [03]

Cuando se prevé que una actividad va a incidir en un factor ambiental, éste se señala en la celda de cruce, describiéndose en términos de su magnitud e importancia [04].

Representan métodos ampliamente usados en los procesos de EIA, puesto que han sido diseñados para ser aplicados a cualquier tipo de proyectos, por lo que son muy populares.

\subsection{Métodos de indicadores}

Se utilizan para valorar características específicas o integradas de factores medioambientales o recursos, como medio para describir los ambientes afectados, así como la predicción y evaluación de impactos. Los índices numéricos o descriptivos se han desarrollado como una medida de la vulnerabilidad del Medio Ambiente y los recursos a la contaminación u otras acciones humanas y han probado su utilidad en la comparación de localizaciones para una actividad propuesta. Sobre estas bases, pueden formularse las medidas para minimizar los impactos ambientales e incluir controles. [02], [01]

Ejemplos de métodos de indicadores son los siguientes:

- Método de Holmes [05]

- Método de la Universidad de Georgia [06]

- Método de Hill-Schechter [07]

- Método de Fisher-Davis [08]

- Procesos de Monitorización [02] [01]

\subsection{Metodología general para la evaluación de impacto ambiental}

El Estudio de Impacto Ambiental (EsIA), es la descripción pormenorizada de las características de un proyecto de obra o actividad que se pretende llevar a cabo, incluyendo su tecnología, que se presenta para su aprobación en el marco del proceso de evaluación de impacto ambiental. Debe proporcionar antecedentes fundados para la predicción, identificación e interpretación del impacto ambiental del proyecto y describir las acciones que se ejecutarán para impedir o minimizar los efectos adversos, así como el programa de monitoreo que se adoptará [03].

EI EsIA constituye un elemento analítico que interviene de manera esencial en cuanto a dar información en el procedimiento administrativo que es la EIA, y que culmina con la Declaración de Impacto Ambiental, la cual debe presentar la "realidad objetiva, para conocer en qué medida repercutirá sobre el entorno la puesta en marcha de un proyecto, obra o actividad" [03]. Además es un proceso complejo, puesto que es realizado por un equipo interdisciplinario, por lo que hay que homogeneizar las predicciones de cada uno de los miembros del equipo, así como la dificultad que representa predecir el efecto conjunto del proyecto sobre el Medio Ambiente, a partir de efectos parciales difíciles de predecir y debe realizarse de forma científicamente válida [09], [10].

La Metodología General consta de dos grandes fases, la Valoración Cualitativa y la Valoración Cuantitativa. 


\subsubsection{Valoración Cualitativa}

En la fase de valoración cualitativa se busca obtener una estimación de los posibles efectos que provocará en el entorno la realización del proyecto mediante una descripción lingüística de sus propiedades. Los distintos expertos clasifican ciertas variables con etiquetas tales como baja, media, etc. para obtener un conocimiento cualitativo del impacto ambiental.

La metodología puede resumirse en los siguientes pasos:

- Describir el medio como un conjunto de factores ambientales afectados por el proyecto o actividad en estudio.

- Describir el proyecto o actividad evaluada como un conjunto de acciones básicas, perfectamente caracterizadas.

- Identificar los impactos que cada acción definida tiene sobre cada factor ambiental identificado.

- Caracterizar cada impacto mediante la estimación de su importancia.

- Analizar la importancia global de la actividad sobre el medio, a partir de las importancias caracterizadas anteriormente.

\subsubsection{Identificación de los factores ambientales.}

El entorno se conforma por un conjunto de elementos interrelacionados, su estudio como un todo resulta muy complejo, por lo que es necesaria una modelación simplificada. Por esta razón se divide en Sistemas Ambientales, estos a su vez en Subsistemas Ambientales, los cuales se dividen en Componentes Ambientales, que finalmente se dividen en Factores Ambientales (Figura 1). Según sea el proyecto, esta división puede simplificarse, reduciendo los niveles de división.

A cada factor medioambiental se asigna su medida de importancia relativa al entorno, medida en unidades de importancia (UIP), la cual se utiliza para efectuar ponderaciones en las estimaciones globales de los impactos. En la determinación de los factores ambientales, y de la importancia asignada a cada uno, deben tenerse en cuenta ciertos criterios básicos (Figura 2):

- Los factores deben ser representativos del entorno, relevantes, excluyentes entre sí, y exhaustivos.

- Los factores deben ser fácilmente identificables, $\mathrm{y}$ fácilmente cuantificables.
Figura 1. Jerarquía de Identificación de factores ambientales.

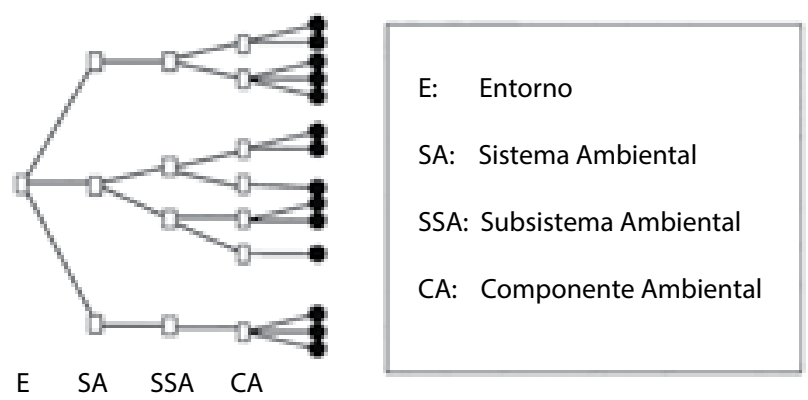

Figura 2. Ejemplo de Factores Ambientales y Unidades de Importancia asignadas.

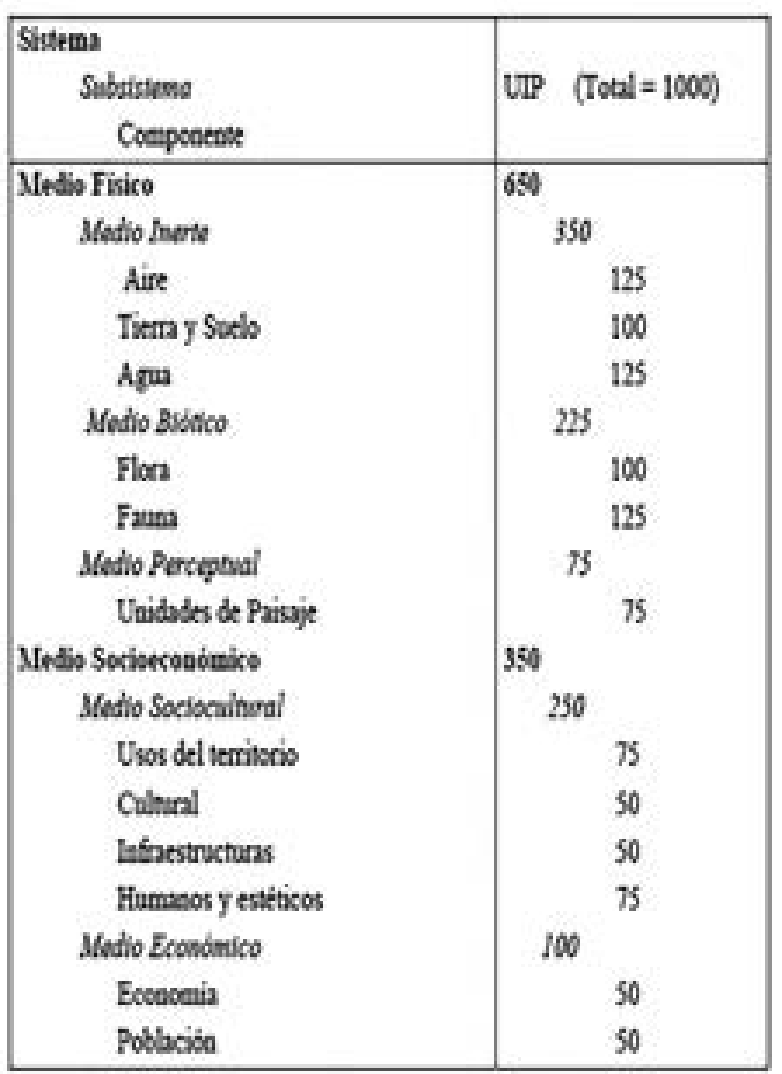

Las UIP que se asignan a cada factor permitirán realizar ponderaciones de los efectos globales; para facilitar esta tarea, así como para facilitar la interpretación de las UIP, se establece la condición de que la suma de las importancias de los factores debe ser 1000. 


\section{Identificación de las acciones del proyecto}

El proyecto que se está evaluando se modela como un conjunto de Acciones, que pueden agruparse en Actividades, y estas a su vez en Situaciones. Muchas veces se desea confrontar varias opciones del mismo proyecto, con el fin de seleccionar aquella de menor impacto al medio; es usual agrupar cada opción como una situación y realizar las comparaciones, para determinar el impacto real de la ejecución proyecto (Figura 3). Teniendo en cuenta las características del proyecto, pueden ser reducidos los niveles de división.

Figura 3. Identificacion de las acciones del proyecto.

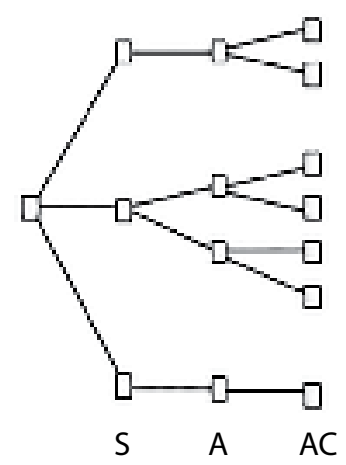

AE: Actuación sobre el Entorno

S: Situaciones

A: Actividades

AC: Acciones

\section{Identificación de los efectos de las acciones del proyecto sobre los factores}

Una vez determinados factores y acciones, se identifican los efectos que estas acciones provocan sobre los factores del Medio Ambiente. Los expertos del equipo interdisciplinar apropiado, son los encargados de realizar esta identificación, que queda materializada en la Matriz de Importancia del proyecto, cuya estructura se muestra en la tabla 1. Los factores están representados en las filas y en las columnas se representan las acciones. En la celda ij de la Matriz se encuentra la Importancia lij del impacto que la acción Aj provoca sobre el factor $\mathrm{Fi}$, la metodología para la determinación de la importancia se describirá en el apartado siguiente. La fila y la columna marcadas como Totales son empleadas para agregar información acerca de una determinada acción o factor respectivamente, según se explicará posteriormente.

\section{DETERMINACIÓN DE LA IMPORTANCIA DE LOS EFECTOS}

La importancia de un impacto es la medida cualitativa del mismo, que se obtiene a partir del grado de incidencia de la alteración producida, y de una caracterización del efecto que responde a una serie de atributos tales como extensión, tipo de efecto, plazo de manifestación, entre otros. Según propone Conesa [03]. La importancia de los impactos se calcula con la ecuación:

$$
\begin{gathered}
I= \pm(3 I+2 E X+M O+P E+R V+S I+A C+E F+ \\
P R+R B)
\end{gathered}
$$

En la Tabla 2, se indican los valores numéricos correspondientes a las variables, según su valoración cualitativa.

Cada Impacto podrá clasificarse de acuerdo a su importancia I como:

- Irrelevante o Compatible: $0 \leq \mathbf{I}<25$

- Moderado: $25 \leq \mathrm{I}<50$

- Severo: $50 \leq \mathrm{I}<75$

- Crítico: $I \geq 75$

Como puede observarse la valoración cualitativa es en realidad una descripción cuantitativa basada en números enteros, pues la importancia se calcula cuantitativamente asignando números enteros a cada una de las etiquetas recogidas en la Figura 4.

\begin{tabular}{|c|c|c|c|c|c|}
\hline \multicolumn{2}{|c|}{ Factores } & \multicolumn{3}{|c|}{ Acciones } & Totales \\
\hline & UIP & $A_{1}$ & $A_{k}$ & $A_{w}$ & \\
\hline$F_{1}$ & $\mathbf{P}_{1}$ & $\mathrm{I}_{\mathrm{U}}$ & $\mathrm{I}_{\mathrm{kk}}$ & $I_{\text {la }}$ & \\
\hline$F_{i}$ & $P_{i}$ & $I_{i l}$ & $\underline{I}_{\mathbf{i k}}$ & $\mathrm{I}_{\mathrm{im}}$ & \\
\hline$F_{n}$ & $P_{n}$ & $I_{n l}$ & $I_{\text {劣 }}$ & $I_{n=}$ & \\
\hline & & & & & \\
\hline
\end{tabular}

Tabla 1. Matriz de Identificación de Impactos 
Figura 4. Variables para la determinación de la importancia de los impactos

Naturaleza: Carácter beneficioso o perjudicial del efecto sobre el factor considerado.

Intensidad: Grado de incidencia de la acción sobre el factor, en el ámbito específico que actúa.

Extensión: Área de influencia teórica del impacto en relación con el entorno del proyecto (\% del área, respecto al entorno, en que se manifiesta el efecto). Si la acción produce un impacto muy bien localizado, se considerará que el impacto tiene un carácter puntual; en caso contrario se considerará total, teniendo gradaciones intermedias parcial y extenso.

Momento: Plazo de manifestación del impacto, tiempo que transcurre entre la aparición de la acción y el comienzo del efecto sobre el factor considerado. Generalmente se expresa en años.

Persistencia: Tiempo supuesto de permanencia del efecto desde su aparición. Una vez transcurrido ese lapso el factor afectado retornaría a las condiciones iniciales previa a la acción por medios naturales, o mediante la introducción de medidas correctoras.

Reversibilidad: Posibilidad de reconstrucción del factor afectado por el proyecto, es la posibilidad de retorno del factor por medios naturales a las condiciones que tenía antes de la ocurrencia de la acción.

Recuperabilidad: Posibilidad de reconstrucción, total o parcial, del factor afectado como consecuencia del proyecto, es decir, la posibilidad de retornar a las condiciones previas a la actuación, por medio de la intervención humana.

Sinergia: Este atributo contempla el reforzamiento de dos o más efectos simple. La componente total de la manifestación de los efectos simples, provocados por acciones que actúan simultáneamente es superior a la que cabría esperar de la manifestación de efectos cuando las acciones actúan por separado.

Acumulación: Incremento de la manifestación de un efecto, cuando persiste de forma continuada la acción que lo genera.

Efecto: Forma de manifestación del efecto sobre un factor como consecuencia de una acción.

Periodicidad: Regularidad de manifestación del efecto.

\section{Análisis Cualitativo Global}

Una vez conformada la Matriz de Importancia con cada una de las importancias de los impactos, se analiza el impacto del proyecto en su totalidad; preliminarmente se efectúa una depuración de la Matriz de Importancia eliminando aquellos impactos:

- Que resulten irrelevantes. Tienen una importancia por debajo de cierto valor umbral.

- Que se presenten sobre factores para los que no se dispone de un indicador adecuado.

- Que resulten extremadamente severos y por tanto necesiten un tratamiento especial.

Seguidamente se procede a la Valoración Cualitativa del Impacto Ambiental Total. Para obtener este indicador se realiza el análisis numérico de la Matriz de Importancia depurada, consistente en sumas y sumas ponderadas por UIP; estas sumas se realizan por filas y por columnas. Nuevamente estamos ante un proceso cuantitativo basado en números enteros, cuando lo que se quiere obtener es una valoración cualitativa.

Las sumas por columnas permiten identificar las acciones más agresivas (valores altos negativos), las poco agresivas (valores bajos negativos) y las beneficiosas (valores positivos); así como las sumas por filas, permiten identificar los factores más afectados por el proyecto, ya sea positiva o negativamente. Si se comparan estos resultados en situaciones diferentes del proyecto se pueden analizar varias alternativas del mismo.

A continuación se muestran algunos indicadores utilizados con el fin de estimar elimpacto conjunto de varios efectos. Se ha supuesto que la matriz tiene $n$ factores, $m$ acciones, y donde lij es la importancia del impacto de la acción $A j$ sobre el factor $F i$, cuya importancia relativa al entorno es $\mathrm{Pi}$.

- Importancia de los efectos debidos a la acción Aj:

$$
I_{A_{j}}=\sum_{i=1}^{n} I_{i j}
$$

- Importancia de los efectos sufridos por el factor Fi:

$$
I_{F_{i}}=\sum_{i=1}^{m} I_{b j}
$$


Tabla 2. Valoración de las Variables para el Cálculo de la Importancia del Impacto [03]

\begin{tabular}{|c|c|c|c|}
\hline NATURALEZA & & INTENSIDA & \\
\hline Impacto beaeficioso & + & Baja & 1 \\
\hline Impacto Perjudicial & - & Media & 2 \\
\hline & & & 4 \\
\hline & & Muy Alta & 8 \\
\hline & & Total & 12 \\
\hline EXTENSION (EX) & & MOMENTO & \\
\hline Puntual & 1 & Largo Plazo & 1 \\
\hline Parcial & 2 & Medio Plazo & 2 \\
\hline Extenso & 4 & Inmediato & 4 \\
\hline Total & 8 & Critico & $(+4)$ \\
\hline Critica & $(+4)$ & & \\
\hline PERSISTENCLA (PE) & & REVERSIBILID. & \\
\hline Fugaz & 1 & Corto Plazo & 1 \\
\hline Temporal & 2 & Medio Plazo & 2 \\
\hline Permanente & 4 & Inreversible & 4 \\
\hline SINERGIA (SI) & & ACUMULACIÓ & \\
\hline Sin Sinergismo & 1 & Simple & 1 \\
\hline Sintergico & 2 & Acumulativo & 4 \\
\hline Muy Sinérgico & 4 & & \\
\hline EFECTO (EF) & & PERIODICIDA & \\
\hline Indirecto (Secundario) & 1 & Irregular o aperiódico y discontinuo & 1 \\
\hline Directo & 4 & Periódico & 2 \\
\hline & & Contiavo & 4 \\
\hline RECUPERABIIIDAD (RB) & & IMPORTANC & \\
\hline Recuperable de manera inmediata & 1 & & \\
\hline Recuperable a medio plazo & 2 & & \\
\hline Mitigable & 4 & $\mathbf{I}= \pm(3 \mathrm{I}+2 \mathrm{EX}+\mathrm{MO}+\mathrm{PE}+\mathrm{RV}+$ & $(E F+P R+R B)$ \\
\hline Irrocuperable & 8 & & \\
\hline
\end{tabular}

- Importancia relativa al entorno de los efectos debidos a la acción Aj:

$$
I_{R-A_{j}}=\sum_{i=1}^{n} P_{i} I_{i j}
$$

- Importancia relativa al entorno de los efectos sufridos por el factor Fj:

$$
I_{R-F_{i}}=\sum_{j=1}^{m} P_{i} I_{i j}=P_{i} \sum_{j=1}^{m} I_{i j}
$$

- Importancia Total del proyecto:

$$
I_{T}=\sum_{i=1}^{n} \sum_{j=1}^{m} I_{j}
$$

- Importancia Total del proyecto relativa al entorno:

$$
I_{R-T}=\sum_{i=1}^{n} \sum_{j=1}^{m} P_{i} I_{i}
$$

\section{Valoración Cuantitativa}

Los resultados obtenidos en la Valoración Cualitativa son un complemento necesario para el desarrollo de la etapa de Valoración Cuantitativa, puesto que en esta fase se realizan estudios técnicos más detallados que permiten predecir numéricamente cada uno de los impactos individuales (a diferencia de la predicción lingüística obtenida en la fase anterior), y que posteriormente se agrupará para obtener una predicción numérica del impacto total.

La predicción numérica se transforma en las variables Calidad Ambiental y Valor Ambiental, que son intangibles y adimensionales, y por estas características, deben tratarse de manera cualitativa; sin embargo, esta metodología no brinda herramientas para ello.

\section{INDICADORES AMBIENTALES Y MAGNITUD DE LOS IMPACTOS}

Un indicador de un factor ambiental es una variable que permite medir al factor. Las unidades de medida de cada indicador están determinadas 
por el propio indicador, por lo que cada factor será medido en unidades diferentes, consecuentemente, no pueden realizarse comparaciones entre dos factores tomando como base sus indicadores.

La Magnitud de un impacto es la estimación cuantitativa del efecto que éste tendrá sobre el factor ambiental, es el valor que se espera tome el indicador del factor. La magnitud del impacto generalmente se registra en la Matriz de Magnitudes.La magnitud del impacto total recibido por un factor es la agregación de las magnitudes de los impactos que provocan las diferentes acciones del proyecto sobre dicho factor, es decir:

Mi= Agi $($ Mi1 , ...,Mij , ..,Mim)

donde Mi es la magnitud del impacto total recibido por el factor $\mathrm{Fi}$, Mij la magnitud del impacto producido por la acción Aj sobre el Factor Fi, Agi es la función de agregación del factor $F i$, y se han supuesto $m$ acciones impactantes. En la tabla 3, se muestran algunos ejemplos de funciones de agregación, pues esta función depende del factor considerado.

Tabla 3. Funciones de agregación

\begin{tabular}{|c|c|}
\hline Agregación & $M_{i}=A g_{i}\left(M_{a}, \cdots, M_{i}, \ldots, M_{i=}\right)$ \\
\hline Sin sinergia & $M_{i}=\sum_{j=1}^{m} M_{i j}$ \\
\hline Con sinergia lineal & $\begin{array}{l}M_{i}=\sum_{j=1}^{m} M_{i j}+\sum_{k=j+1}^{m} S_{i j}\left(M_{i j}+M_{i k}\right) \\
\mathrm{S}_{i j} \text { es el coeficiente de sinergia del factor } \mathrm{F}_{i}\end{array}$ \\
\hline Con sinergia potencial & $\begin{array}{l}\qquad M_{i}=K^{r-1} \sum_{j=1}^{m} M_{y} \\
\mathrm{~K} \text { es el coeficiente de sinergia }(\mathrm{K}>1) \text { y } \mathrm{r} \text { es el número } \\
\text { de acciones impactantes }(\mathrm{r}=\mathrm{n}) \text {. Si } \mathrm{K}>1 \text { existe sinergia } \\
\text { positiva, en caso contrario se trata de sinergia } \\
\text { negativa, o debilitamiento. }\end{array}$ \\
\hline Logaritmica & $M_{f}=10 \log _{10}\left[\sum_{j=f}^{m} 10\left(M_{n} / 10\right)\right]$ \\
\hline
\end{tabular}

Tabla 4. Categoría de diagnóstico

\begin{tabular}{|c|c|}
\hline Categoría de diagnostico & $\begin{array}{c}\text { Rango de nivel de } \\
\text { importancia }\end{array}$ \\
\hline IRREVELANTE & $0 \leq \mathrm{I}<25$ \\
MODERADO & $25 \leq \mathrm{I}<50$ \\
SEVERO & $50 \leq \mathrm{I}<75$ \\
CRITICO & $\mathrm{I} \geq 75$ \\
\hline
\end{tabular}

Tabla 5. Categoría de medidas de mitigación

\begin{tabular}{|c|c|}
\hline $\begin{array}{c}\text { Categoría de medida de } \\
\text { mitigación }\end{array}$ & $\begin{array}{c}\text { Rango de nivel de } \\
\text { importancia }\end{array}$ \\
\hline IRREVELANTE & $0 \leq \mathrm{I}<25$ \\
MODERADO & $25 \leq \mathrm{I}<50$ \\
SEVERO & $50 \leq \mathrm{I}<75$ \\
CRITICO & $\mathrm{I} \geq 75$ \\
\hline
\end{tabular}




\section{DESARROLLO DE LOS CRITERIOS DE EVALUACION}

7.1. En la Tabla 6 mostraremos los resultados y pesos a considerar para una evaluación, según los rangos mostrados en la Tabla 4 de ccategoría de diagnóstico

Tabla 6. Resultados y pesos

\begin{tabular}{|c|c|c|c|c|c|c|}
\hline \multirow{3}{*}{ CAT } & \multirow{3}{*}{ C.A } & \multirow{3}{*}{ PARAMETROS } & \multicolumn{4}{|c|}{ RESULTADO } \\
\hline & & & $0 \leq 1<25$ & $25 \leq 1<50$ & $50 \leq 1<75$ & $I \geq 75$ \\
\hline & & & IRRELEVANTE & MODERADO & SEVERO & CRITICO \\
\hline \multirow{8}{*}{$\begin{array}{l}0 \\
\frac{0}{\omega} \\
\frac{\omega}{L}\end{array}$} & \multirow[b]{2}{*}{$\stackrel{0}{\stackrel{0}{\alpha}}$} & $\begin{array}{l}\text { CALIDAD } \\
\text { DEL AIRE }\end{array}$ & $\begin{array}{l}\text { Calidad de aire casi } \\
\text { o sin alteración/ sin } \\
\text { plan de acción }\end{array}$ & $\begin{array}{l}\text { Efecto moderado } \\
\text { de calidad de } \\
\text { aire /acciones } \\
\text { preventivas }\end{array}$ & $\begin{array}{l}\text { Efecto severo de } \\
\text { calidad de aire / } \\
\text { acciones mitigantes }\end{array}$ & $\begin{array}{l}\text { Efecto critico } \\
\text { de calidad de } \\
\text { aire /acciones } \\
\text { compensatorias }\end{array}$ \\
\hline & & $\begin{array}{c}\text { RUIDOS Y } \\
\text { VIBRACIONES }\end{array}$ & $\begin{array}{l}\text { Poca o sin emisión } \\
\text { de ruidos y } \\
\text { vibraciones / Sin } \\
\text { plan de acción }\end{array}$ & $\begin{array}{l}\text { Efecto moderado } \\
\text { de ruidos y } \\
\text { vibraciones / } \\
\text { acciones } \\
\text { preventivas }\end{array}$ & $\begin{array}{l}\text { Efecto severo de } \\
\text { ruidos y vibraciones } \\
\text { lacciones } \\
\text { mitigantes }\end{array}$ & $\begin{array}{l}\text { Efecto critico de } \\
\text { ruidos y vibraciones } \\
\text { /acciones } \\
\text { compensatorias }\end{array}$ \\
\hline & \multirow{3}{*}{$\frac{\circ}{\frac{0}{0}}$} & $\begin{array}{l}\text { FISIOLOGÍA/ } \\
\text { GEOMORFO }\end{array}$ & $\begin{array}{l}\text { Fisiología casi o sin } \\
\text { alteración/ sin plan } \\
\text { de acción }\end{array}$ & $\begin{array}{l}\text { Efecto moderado } \\
\text { de fisiología / } \\
\text { acciones } \\
\text { preventivas }\end{array}$ & $\begin{array}{l}\text { Efecto severo de } \\
\text { fisiología /acciones } \\
\text { mitigantes }\end{array}$ & $\begin{array}{l}\text { Efecto critico de } \\
\text { fisiología /acciones } \\
\text { compensatorias }\end{array}$ \\
\hline & & $\begin{array}{l}\text { CALIDAD DE } \\
\text { SUELO }\end{array}$ & $\begin{array}{l}\text { Calidad de } \\
\text { suelo casi o sin } \\
\text { alteración/ sin plan } \\
\text { de acción }\end{array}$ & $\begin{array}{l}\text { Efecto moderado } \\
\text { de calidad de } \\
\text { suelo /acciones } \\
\text { preventivas }\end{array}$ & $\begin{array}{l}\text { Efecto severo de } \\
\text { calidad de suelo / } \\
\text { acciones mitigantes }\end{array}$ & $\begin{array}{l}\text { Efecto critico } \\
\text { de calidad de } \\
\text { suelo /acciones } \\
\text { compensatorias } \\
\end{array}$ \\
\hline & & $\begin{array}{l}\text { CAPACIDAD DE } \\
\text { USO }\end{array}$ & $\begin{array}{l}\text { Capacidad de } \\
\text { uso casi o sin } \\
\text { alteración/ sin plan } \\
\text { de acción }\end{array}$ & $\begin{array}{l}\text { Efecto moderado } \\
\text { de capacidad de } \\
\text { uso /acciones } \\
\text { preventivas }\end{array}$ & $\begin{array}{l}\text { Efecto severo de } \\
\text { capacidad de uso / } \\
\text { acciones mitigantes }\end{array}$ & $\begin{array}{l}\text { Efecto critico de } \\
\text { capacidad de } \\
\text { uso /acciones } \\
\text { compensatorias }\end{array}$ \\
\hline & \multirow{3}{*}{ 芩 } & $\begin{array}{l}\text { CALIDAD DEL } \\
\text { AGUA SUPERF }\end{array}$ & $\begin{array}{l}\text { Calidad de agua } \\
\text { superficial casi o } \\
\text { sin alteración/ sin } \\
\text { plan de acción }\end{array}$ & $\begin{array}{l}\text { Efecto moderado } \\
\text { de calidad de } \\
\text { agua superficial } \\
\text { /acciones } \\
\text { preventivas }\end{array}$ & $\begin{array}{l}\text { Efecto severo } \\
\text { de calidad de } \\
\text { agua superficial / } \\
\text { acciones mitigantes }\end{array}$ & $\begin{array}{l}\text { Efecto critico } \\
\text { de calidad de } \\
\text { agua superficial } \\
\text { /acciones } \\
\text { compensatorias }\end{array}$ \\
\hline & & $\begin{array}{l}\text { CALIDAD AGUA } \\
\text { SUBTERRÁNEA }\end{array}$ & $\begin{array}{l}\text { calidad de agua } \\
\text { subterránea casi o } \\
\text { sin alteración/ sin } \\
\text { plan de acción }\end{array}$ & $\begin{array}{l}\text { Efecto moderado } \\
\text { de calidad de } \\
\text { agua subterránea } \\
\text { /acciones } \\
\text { preventivas }\end{array}$ & $\begin{array}{l}\text { Efecto severo de } \\
\text { calidad de agua } \\
\text { subterránea / } \\
\text { acciones mitigantes }\end{array}$ & $\begin{array}{l}\text { Efecto critico de } \\
\text { calidad de agua } \\
\text { subterránea / } \\
\text { acciones } \\
\text { compensatorias }\end{array}$ \\
\hline & & $\begin{array}{l}\text { DISMINUCIÓN } \\
\text { DEL RECURSO } \\
\text { HIDRICP }\end{array}$ & $\begin{array}{l}\text { Cantidad de } \\
\text { recurso hídrico casi } \\
\text { o sin alteración/ sin } \\
\text { plan de acción }\end{array}$ & $\begin{array}{l}\text { Efecto moderado } \\
\text { de disminución } \\
\text { de recurso } \\
\text { hídrico /acciones } \\
\text { preventivas }\end{array}$ & $\begin{array}{l}\text { Efecto severo de } \\
\text { disminución de } \\
\text { recurso hídrico/ } \\
\text { acciones mitigantes }\end{array}$ & $\begin{array}{l}\text { Efecto critico } \\
\text { de disminución } \\
\text { de recurso } \\
\text { hídrico /acciones } \\
\text { compensatorias }\end{array}$ \\
\hline \multirow{2}{*}{ 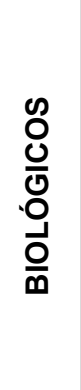 } & & $\begin{array}{l}\text { DIVERSIDAD Y } \\
\text { ABUNDANCIA }\end{array}$ & $\begin{array}{l}\text { Diversidad y } \\
\text { abundancia de } \\
\text { fauna casi o sin } \\
\text { alteración/ sin plan } \\
\text { de acción }\end{array}$ & $\begin{array}{l}\text { Efecto moderado } \\
\text { en la diversidad } \\
\text { y abundancia de } \\
\text { Fauna /acciones } \\
\text { preventivas }\end{array}$ & $\begin{array}{l}\text { Efecto severo en } \\
\text { la diversidad y } \\
\text { abundancia de } \\
\text { Fauna/acciones } \\
\text { mitigantes }\end{array}$ & $\begin{array}{l}\text { Efecto critico en } \\
\text { la diversidad y } \\
\text { abundancia de } \\
\text { Fauna /acciones } \\
\text { compensatorias }\end{array}$ \\
\hline & & $\begin{array}{l}\text { ALTERACIÓN } \\
\text { DEL HÁBITAT }\end{array}$ & $\begin{array}{l}\text { Hábitat de } \\
\text { fauna casi o sin } \\
\text { alteración/ sin plan } \\
\text { de acción }\end{array}$ & $\begin{array}{l}\text { Efecto moderado } \\
\text { de alteración } \\
\text { de hábitat de } \\
\text { Fauna /acciones } \\
\text { preventivas }\end{array}$ & $\begin{array}{l}\text { Efecto severo } \\
\text { de alteración } \\
\text { de hábitat de } \\
\text { Fauna /acciones } \\
\text { preventivas }\end{array}$ & $\begin{array}{l}\text { Efecto critico } \\
\text { de alteración } \\
\text { de hábitat de } \\
\text { Fauna /acciones } \\
\text { compensatorias }\end{array}$ \\
\hline
\end{tabular}


... Viene de la pág. anterior

\begin{tabular}{|c|c|c|c|c|c|c|}
\hline \multirow{4}{*}{ 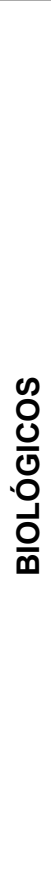 } & $\underset{\substack{\pi \\
\frac{\pi}{2}}}{\stackrel{\pi}{\leftarrow}}$ & $\begin{array}{l}\text { ESPECIES } \\
\text { PROTEGIDAS }\end{array}$ & $\begin{array}{l}\text { Especies } \\
\text { protegidas de } \\
\text { fauna casi o sin } \\
\text { alteración/ sin plan } \\
\text { de acción }\end{array}$ & $\begin{array}{l}\text { Efecto moderado } \\
\text { de especies } \\
\text { protegidas de } \\
\text { Fauna /acciones } \\
\text { preventivas }\end{array}$ & $\begin{array}{l}\text { Efecto severo de } \\
\text { especies protegidas } \\
\text { de Fauna /acciones } \\
\text { preventivas }\end{array}$ & $\begin{array}{l}\text { Efecto critico de } \\
\text { especies protegidas } \\
\text { de Fauna /acciones } \\
\text { compensatorias }\end{array}$ \\
\hline & \multirow{3}{*}{$\frac{\text { 민 }}{\frac{1}{4}}$} & $\begin{array}{l}\text { DIVERSIDAD Y } \\
\text { ABUNDANCIA }\end{array}$ & $\begin{array}{l}\text { Diversidad y } \\
\text { abundancia de } \\
\text { flora casi o sin } \\
\text { alteración/ sin plan } \\
\text { de acción }\end{array}$ & $\begin{array}{l}\text { Efecto moderado } \\
\text { de diversidad y } \\
\text { abundancia de } \\
\text { flora /acciones } \\
\text { preventivas }\end{array}$ & $\begin{array}{l}\text { Efecto severo } \\
\text { de diversidad y } \\
\text { abundancia de } \\
\text { flora /acciones } \\
\text { preventivas }\end{array}$ & $\begin{array}{l}\text { Efecto critico } \\
\text { de diversidad y } \\
\text { abundancia de } \\
\text { flora/acciones } \\
\text { compensatorias }\end{array}$ \\
\hline & & $\begin{array}{l}\text { ALTERACIÓN } \\
\text { DEL HABITAT }\end{array}$ & $\begin{array}{l}\text { Hábitat de } \\
\text { flora casi o sin } \\
\text { alteración/ sin plan } \\
\text { de acción }\end{array}$ & $\begin{array}{l}\text { Efecto moderado } \\
\text { de alteración } \\
\text { de hábitat de } \\
\text { Fauna /acciones } \\
\text { preventivas }\end{array}$ & $\begin{array}{l}\text { Efecto severo } \\
\text { de alteración } \\
\text { de hábitat de } \\
\text { Fauna /acciones } \\
\text { preventivas }\end{array}$ & $\begin{array}{l}\text { Efecto critico } \\
\text { de alteración } \\
\text { de hábitat de } \\
\text { Fauna /acciones } \\
\text { compensatorias }\end{array}$ \\
\hline & & $\begin{array}{l}\text { ESPECIES } \\
\text { PROTEGIDAS }\end{array}$ & $\begin{array}{l}\text { Especies } \\
\text { protegidas de } \\
\text { flora casi o sin } \\
\text { alteración/ sin plan } \\
\text { de acción }\end{array}$ & $\begin{array}{l}\text { Efecto moderado } \\
\text { de especies } \\
\text { protegidas de } \\
\text { Fauna /acciones } \\
\text { preventivas }\end{array}$ & $\begin{array}{l}\text { Efecto severo de } \\
\text { especies protegidas } \\
\text { de Fauna /acciones } \\
\text { mitigantes }\end{array}$ & $\begin{array}{l}\text { Efecto critico de } \\
\text { especies protegidas } \\
\text { de Fauna /acciones } \\
\text { compensatorias }\end{array}$ \\
\hline
\end{tabular}

7.2. En la Tabla 7 mostraremos las mmedidas de mitigación a considerar en una evaluación, según los rangos mostrados en la Tabla 5 de categoría de medidas de mitigación.

Tabla 7. Categoría de medidas de mitigación

\begin{tabular}{|c|c|c|c|c|c|c|}
\hline \multirow{3}{*}{ CAT } & \multirow{3}{*}{ C.A } & \multirow{3}{*}{ PARÁMETROS } & \multicolumn{4}{|c|}{ RESULTADO } \\
\hline & & & $0 \leq 1<25$ & $25 \leq 1<50$ & $50 \leq 1<75$ & $I \geq 75$ \\
\hline & & & IRRELEVANTE & MODERADO & SEVERO & CRITICO \\
\hline \multirow{3}{*}{$\begin{array}{l}\mathscr{0} \\
\frac{0}{\mathscr{W}}\end{array}$} & & $\begin{array}{l}\text { CALIDAD } \\
\text { DEL AIRE }\end{array}$ & Sin plan de acción & $\begin{array}{l}\text { Dar instructivos de } \\
\text { trabajo }\end{array}$ & Normas & $\begin{array}{l}\text { Programas de } \\
\text { monitoreo }\end{array}$ \\
\hline & $\stackrel{\varrho}{\stackrel{2}{<}}$ & $\begin{array}{c}\text { RUIDOS Y } \\
\text { VIBRACIONES }\end{array}$ & Sin plan de acción & $\begin{array}{l}\text { Normas de } \\
\text { seguridad industrial } \\
\text { y ocupacional/ } \\
\text { Mantenimiento de } \\
\text { equipos y vehículos }\end{array}$ & $\begin{array}{l}\text { Mantenimiento } \\
\text { de equipos y } \\
\text { vehículos/ } f \text { Se } \\
\text { elaborará un } \\
\text { programa de } \\
\text { mantenimiento } \\
\text { mecánico } \\
\text { funcionamiento } \\
\text { óptimo de los } \\
\text { equipos con } \\
\text { emisiones } \\
\text { dentro de límites } \\
\text { aceptables. } f\end{array}$ & $\begin{array}{l}\text { Programas de } \\
\text { monitoreo }\end{array}$ \\
\hline & $\frac{\circ}{\mathscr{0}}$ & $\begin{array}{l}\text { FISIOLOGIA/ } \\
\text { GEOMORFO }\end{array}$ & Sin plan de acción & $\begin{array}{l}\text { Planificación de } \\
\text { actividades }\end{array}$ & $\begin{array}{l}\text { Conservación de } \\
\text { suelos }\end{array}$ & $\begin{array}{l}\text { Plan de Manejo } \\
\text { de } \\
\text { Residuos } \\
\text { Peligrosos }\end{array}$ \\
\hline
\end{tabular}

Continúa... 
... Viene de la pág. anterior

\begin{tabular}{|c|c|c|c|c|c|c|}
\hline \multirow{5}{*}{ 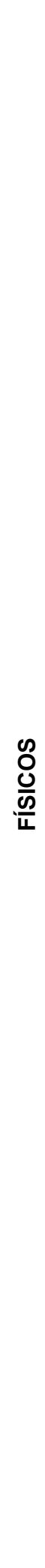 } & \multirow[t]{2}{*}{$\frac{\circ}{\frac{0}{0}}$} & $\begin{array}{l}\text { CALIDAD DE } \\
\text { SUELO }\end{array}$ & Sin plan de acción & $\begin{array}{l}\text { Dar instructivos } \\
\text { de trabajo/ Los } \\
\text { caminos de acceso } \\
\text { serán trazados } \\
\text { de manera que } \\
\text { minimice la } \\
\text { perturbación del } \\
\text { terreno. }\end{array}$ & Normas & $\begin{array}{l}\text { Programas de } \\
\text { monitoreo }\end{array}$ \\
\hline & & $\begin{array}{c}\text { CAPACIDAD DE } \\
\text { USO }\end{array}$ & Sin plan de acción & $\begin{array}{l}\text { Diseño de } \\
\text { taludes en base } \\
\text { a condiciones } \\
\text { geotécnicas y de } \\
\text { estabilidad }\end{array}$ & $\begin{array}{l}\text { Programas de } \\
\text { manejo de residuo }\end{array}$ & $\begin{array}{l}\text { Programas de } \\
\text { monitoreo de } \\
\text { capacidad de uso }\end{array}$ \\
\hline & \multirow[t]{3}{*}{$\stackrel{\frac{\pi}{5}}{\frac{9}{4}}$} & $\begin{array}{l}\text { CALIDAD DEL } \\
\text { AGUA SUPERF }\end{array}$ & Sin plan de acción & $\begin{array}{l}\text { Planificación de } \\
\text { actividades }\end{array}$ & $\begin{array}{l}\text { Planes de } \\
\text { contingencia de } \\
\text { derrames, incluso } \\
\text { descubrimiento, } \\
\text { contención y } \\
\text { limpieza, }\end{array}$ & $\begin{array}{l}\text { Minimizar la } \\
\text { penetración en } \\
\text { los acuíferos } \\
\text { desde la } \\
\text { superficie } \\
\text { reteniendo los } \\
\text { contaminantes } \\
\text { en pozas } \\
\text { impermeables. } \\
\text { Taponar o sellar } \\
\text { adecuadamente } \\
\text { el pozo mediante } \\
\text { lodo, gel, o } \\
\text { cementos } \\
\text { aprobados u } \\
\text { otros materiales } \\
\text { apropiados } \\
\text { que no causen } \\
\text { daño al medio } \\
\text { ambiente. }\end{array}$ \\
\hline & & $\begin{array}{l}\text { CALIDAD AGUA } \\
\text { SUBTERRÁNEA }\end{array}$ & Sin plan de acción & $\begin{array}{l}\text { Normas de } \\
\text { seguridad industrial, } \\
\text { ocupacional e } \\
\text { instructivos de } \\
\text { trabajo }\end{array}$ & $\begin{array}{l}\text { Diseño de obras de } \\
\text { manejo de aguas } \\
\text { de escorrentía y } \\
\text { drenaje }\end{array}$ & $\begin{array}{l}\text { Programas de } \\
\text { monitoreo }\end{array}$ \\
\hline & & $\begin{array}{l}\text { DISMINUCIÓN } \\
\text { DEL RECURSO } \\
\text { HÍDRICO }\end{array}$ & Sin plan de acción & $\begin{array}{l}\text { Planes de } \\
\text { contingencia ante } \\
\text { derrames/Reducir } \\
\text { o eliminar solidos } \\
\text { suspendidos }\end{array}$ & $\begin{array}{l}\text { Protección de la } \\
\text { calidad de las } \\
\text { aguas }\end{array}$ & $\begin{array}{l}\text { Mitigar } \\
\text { proporcionando } \\
\text { otros suministros } \\
\text { de agua, } \\
\text { recirculando } \\
\text { y empleando } \\
\text { procesos } \\
\text { eficientes en el } \\
\text { uso del agua. Se } \\
\text { deberá investigar } \\
\text { otras fuentes de } \\
\text { suministro }\end{array}$ \\
\hline
\end{tabular}

... Continúa 
... Viene de la pág. anterior

\begin{tabular}{|c|c|c|c|c|c|c|}
\hline \multirow{6}{*}{$\begin{array}{l}\text { o } \\
\text { U. } \\
\text { o } \\
\frac{0}{m}\end{array}$} & \multirow{3}{*}{ 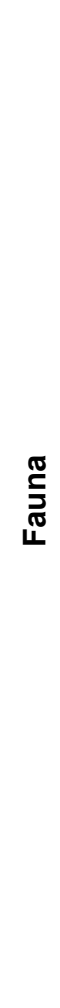 } & $\begin{array}{l}\text { DIVERSIDAD Y } \\
\text { ABUNDANCIA }\end{array}$ & Sin plan de acción & $\begin{array}{l}\text { Limitar areas de } \\
\text { intervención }\end{array}$ & $\begin{array}{l}\text { Dar prioridad a } \\
\text { una investigación, } \\
\text { dirigida a evaluar } \\
\text { el estado, la } \\
\text { tendencia de la } \\
\text { población de los } \\
\text { animales presentes } \\
\text { en el yacimiento. }\end{array}$ & $\begin{array}{l}\text { Recuperar } \\
\text { inmediatamente } \\
\text { las áreas } \\
\text { alteradas a } \\
\text { medida que } \\
\text { se tornan } \\
\text { disponibles } \\
\text { para restaurar } \\
\text { el forraje y el } \\
\text { hábitat. }\end{array}$ \\
\hline & & $\begin{array}{l}\text { ALTERACIÓN } \\
\text { DEL HÁBITAT }\end{array}$ & Sin plan de acción & $\begin{array}{l}\text { Medida de } \\
\text { manejo generales } \\
\text { y educación } \\
\text { ambiental } \\
\text { (construcción y } \\
\text { operación) }\end{array}$ & $\begin{array}{l}\text { Plan de rescate de } \\
\text { fauna. }\end{array}$ & $\begin{array}{l}\text { Reinserción de } \\
\text { fauna propia del } \\
\text { lugar }\end{array}$ \\
\hline & & $\begin{array}{l}\text { ESPECIES } \\
\text { PROTEGIDAS }\end{array}$ & Sin plan de acción & $\begin{array}{l}\text { Mantener vedadas } \\
\text { aquellas especies } \\
\text { sobre las cuales } \\
\text { existen evidencias } \\
\text { de peligro de } \\
\text { extinción. }\end{array}$ & $\begin{array}{l}\text { Aplicar métodos } \\
\text { de cuidado y } \\
\text { vigilancia, con el } \\
\text { fin de minimizar las } \\
\text { alteraciones sobre } \\
\text { la vida animal en } \\
\text { las distintas fases } \\
\text { del sistema de } \\
\text { explotación }\end{array}$ & $\begin{array}{l}\text { Recolonización } \\
\text { de fauna }\end{array}$ \\
\hline & \multirow{3}{*}{$\frac{\pi}{\frac{0}{4}}$} & $\begin{array}{l}\text { DIVERSIDAD Y } \\
\text { ABUNDANCIA }\end{array}$ & Sin plan de acción & $\begin{array}{l}\text { Limitar areas de } \\
\text { intervención }\end{array}$ & $\begin{array}{l}\text { semillero y } \\
\text { enriquecimiento } \\
\text { de cobertura } \\
\text { de herbáceas/ } \\
\text { Inducción de } \\
\text { recolonización }\end{array}$ & $\begin{array}{l}\text { Programas de } \\
\text { revegetación }\end{array}$ \\
\hline & & $\begin{array}{l}\text { ALTERACIÓN } \\
\text { DEL HÁBITAT }\end{array}$ & Sin plan de acción & $\begin{array}{l}\text { Protección de la } \\
\text { flora }\end{array}$ & $\begin{array}{l}\text { plan de rescate } \\
\text { y relocalización } \\
\text { de cactáceas y } \\
\text { arbustos }\end{array}$ & $\begin{array}{l}\text { Reforestación } \\
\text { de la zona } \\
\text { priorizando } \\
\text { árboles y } \\
\text { arbustos del } \\
\text { mismo lugar.. }\end{array}$ \\
\hline & & $\begin{array}{c}\text { ESPECIES } \\
\text { PROTEGIDAS }\end{array}$ & Sin plan de acción & $\begin{array}{l}\text { La exclusión de } \\
\text { sectores para no } \\
\text { intervención, la } \\
\text { conservación de } \\
\text { material genético in } \\
\text { y ex -situ. }\end{array}$ & $\begin{array}{l}\text { implementación } \\
\text { de viveros para } \\
\text { arbustos }\end{array}$ & $\begin{array}{l}\text { La producción } \\
\text { de plantas en } \\
\text { categorías de } \\
\text { conservación y la } \\
\text { reforestación con } \\
\text { estas especies } \\
\text { nativas }\end{array}$ \\
\hline
\end{tabular}

\section{CONCLUSIONES}

1. Los criterios de evaluación de impacto ambiental presentados en las Tablas 6 y 7 , ayudarán a las empresas mineras a que su proyecto de inversión sea favorable para su aprobación por el Ministerio de ambiente.
2. La evaluación de impacto ambiental por ser multidisciplinario, necesita el aporte de diversos profesionales, como biólogos, ingenieros mineros, ingenieros ambientales, meteorólogos etc., que pueda brindar una gama de posibles medidas de mitigación para obtener parámetros que sean aceptados. 


\section{REFERENCIAS BIBLIOGRÁFICAS}

[1] García Leyton, L.A. Aplicación del Análisis Multicriterio en la Evaluación de Impactos Ambientales. Tesis Doctoral Universidad Politécnica de Catalunya 2004.

[2] Canter, L.; Sadler, B. A tool kit for effective environmental impact assessment practice review of methods and perspectives on their application. Asupplementary report of international study of the effectiveness of environmental assessment. International Association for Impact Assessment (IAIA), 1997.

[3] Conesa Fernández, V. Guía Metodológica para la Evaluación de Impacto Ambiental (2da Edición). Editorial Mundi-Prensa, Madrid, 1997

[4] Canter, L. Manual de Impacto Ambiental (2da Edición). Mc Graw-Hill, Madrid,1998.

[5] Holmes, J.C. An ordinal method of evaluation. Urban Studies 9 (1972). 179-191

[6] Odum, Howard T. Use of Energy Diagrams for Environmental Impact Statements.In Proceedings of the Conference Tools for
Coastal Management. Marine Technology Society Washington, D.C. 1972. 197-213.

[7] Hill, M.; Shechter, M. Optimal Goal Achievement in the Development of Outdoor Recreation Facilities. In A.G. Wilson, ed., Urban and Regional Planning. London: Pion, 1971. 110-121

[8] Fischer D.W., Davis G.S. An approach to assessing environmental impacts. Journalof Environmental Management, 1 (1973). 207-227.

[9] Aguiló Alonso, M.;Aramburu, M.P.;Cifuentes, P. et al. Guía para la Elaboración de estudios del Medio Físico: Contenido y Metodología. Ministerio de Medio Ambiente, Madrid, 1998.

[10] Álvarez, C. Sierra, V. Metodología de la Investigación Científica. Centro Manuel F. Gran, Santiago de Cuba, 1995.

[11] http://www.minam.gob.pe/ (visitado 05 de Enero de 2015)

[12] h t t p : / / w w w. t e I e I e y. c o m / revistaperuana/18rabanal-65.pdf (visitado el 07 de Enero de 2015)

[13] http://www.lamineriaentuvida.com.ar/que-esla-mineria/ (visitado el 08 de Enero de 2011. 